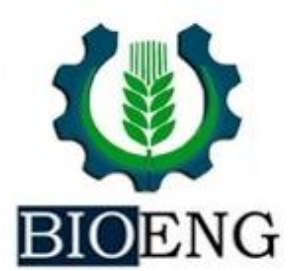

\title{
FILTRAÇÃO LENTA: MAPEAMENTO SISTEMÁTICO DE TESES E DISSERTAÇÕES BRASILEIRAS
}

\author{
A. J. Lima* , D. G. Santos, D. C. G. S. Michelan, L. C. Mendonça
}

UFS - Universidade Federal de Sergipe, São Cristóvão, SE, Brasil

Article history: Received 21 March 2019; Received in revised form 16 May 2019; Accepted 22 May 2019 ; Available online 24 June 2019.

\section{RESUMO}

As primeiras estações de tratamento de água eram constituídas de filtros lentos. A ação de peneiramento juntamente com mecanismos físico-químicos e destacadamente biológicos dotavam este sistema de grande eficiência no tratamento de água, principalmente no que se refere a microrganismos patogênicos. Entretanto, este processo vem sendo substituído por técnicas mais complexas e, em muitos casos, mais caras. Isto se deve em parte pela redução da qualidade da água dos mananciais abastecedores e parte pelo desconhecimento das possibilidades e soluções técnicas para filtração lenta. Dada a situação supracitada, esta pesquisa realizou um mapeamento sistemático da literatura pela busca por teses e dissertações apresentadas aos programas de pós-graduação brasileiros, adotando procedimentos padronizados para este tipo de revisão. O mapeamento sistemático resultou em 29 trabalhos alinhados ao tema de pesquisa, que constituíram o Portifólio Bibliográfico da pesquisa, de maneira que sua análise permitiu, dentre outros: avaliar as publicações por período, instituições que mais estudam o tema, professores envolvidos e suas relações, conhecer os programas potencialmente detentores de trabalhos sobre o tema e ratificar a eficiência dos termos "filtração lenta" e "tratamento de água" na busca por trabalhos sobre filtros lentos.

Palavras-chave: filtros lentos de areia; tratamento de água; revisão da literatura

\section{SLOW FILTRATION: SYSTEMATIC MAPPING OF BRAZILIAN THESES AND DISSERTATIONS}

\begin{abstract}
The first water treatment plants were incorporated slow filters. Screening action together with physico-chemical mechanisms and notably providing this great system biological efficiency in water treatment, mainly with regard to pathogenic micro-organisms. However, this process has been replaced by more complex techniques and, in many cases, more expensive. This is due in part by the reduction of water quality of water sources and providers part by ignorance of the possibilities and technical solutions to slow filtration. Given situation referred to above, this research has conducted a systematic mapping of the literature search for theses and dissertations submitted to the Brazilian graduate programs, adopting standard procedures for this type of review. The systematic mapping resulted in 29 work aligned to the research topic, which constituted the Bibliographical Research Portfolio, so that your analysis has allowed, among others: evaluate the publications per period, institutions that most study the subject, teachers involved and their relationships, meet the programs potentially in possession of
\end{abstract}

\footnotetext{
*eng.civil.andersonlima@gmail.com
} 
papers on the topic and ratify the efficiency of "filtration slow" and "treatment of water" in the search for work on slow filters.

Keywords: slow sand filters, water treatment, review of the literature.

\section{INTRODUÇÃO}

As primeiras experiências com tratamento de água através de filtração em meio granular ocorreram na Escócia e Inglaterra durante o século XIX. O escocês John Gibb construiu um filtro de areia para o tratamento de água utilizada no processo de branqueamento de tecidos, enquanto James Simpson construiu uma estação de tratamento de água por filtração lenta para tratar água que abastecia a cidade de Londres (DI BERNARDO \& DANTAS, 2005).

As primeiras estações de tratamento de água (ETA) eram constituídas somente de filtros lentos que, em geral, operavam com taxa de filtração inferiores a $6 \mathrm{~m}^{3} / \mathrm{m}^{2}$.dia. As águas utilizadas no processo de filtração lenta devem possuir valores baixos de turbidez, cor verdadeira, sólidos suspensos, entre outros, já que normalmente não se emprega coagulação para clarificar a água antes da filtração. Quando não se consegue atender às restrições dos parâmetros supracitados, o que ocorre na atualidade, devido ao decaimento da qualidade das águas dos mananciais pela ação antrópica, é comum utilizar etapas de pré-tratamento comumente constituídas por pré-filtros em pedregulho seguido de filtro lento. Esta configuração de tratamento denomina-se filtração em múltiplas etapas - FiME (DI BERNARDO \& SOBOGAL PAZ, 2008).

No processo de filtração lenta, as impurezas da água são removidas por ação de peneiramento e por uma associação de processos físico-químicos e biológicos. Este último com maior influência, pois durante a filtração há formação da camada biológica denominada schmutzdecke, que funciona como manta biológica na remoção de cor, turbidez e microrganismos patogênicos. Nesta camada, processos microbiológicos como predação, competição, morte natural e necrofagia, promovem a redução de patógenos (RICHTER, 2009; VARGAS, 1999). Além disso, a elevada eficiência de remoção de microrganismos patogênicos na filtração lenta está atrelada a maturidade biológica do meio filtrante, taxas de filtração aplicadas, espessura e características do leito filtrante (HELLER et al., 2004; BRITO et al., 2005).

Apesar dos notáveis benefícios e facilidades de construção, manutenção e operação, o tratamento por filtração lenta está sendo marginalizado por suas dificuldades em tratar águas de baixa qualidade. Souza et al. (2018) realizaram um levantamento e diagnóstico das estações de tratamento que usavam filtração lenta no estado de Santa Catarina e concluíram que o incentivo para substituição da filtração lenta por ETA's convencionais advém: do desejo de experimentar novas tecnologias de tratamento e remediação de problemas corriqueiras, mas solucionáveis no âmbito da filtração lenta; da influência da sazonalidade na qualidade da água bruta alinhadas ao manejo inadequado das nascentes e fontes abastecedoras; e do desconhecimento e despreparo dos envolvidos no tratamento com filtração lenta, suas possiblidades e limitações considerando a qualidade da água bruta e os processos operacionais. Ademais, os autores relatam que a maioria dos problemas identificados na pesquisa poderiam ser facilmente resolvidos caso houvesse operadores preparados minimamente e com mínimo de investimento. De acordo com Di Bernardo (2003), Santa Catarina é o estado brasileiro que mais possui ETA's que aplicam a técnica de filtração lenta.

Assim sendo, este trabalho tem como objetivo analisar a produção de trabalhos sobre filtração lenta pelos 
programas de pós-graduação brasileiros de modo a conhecer os programas de pósgraduação potencialmente detentores de trabalhos relativos ao tema, bem como suas instituições de origem; quantificar as publicações por período; conhecer as instituições que mais estudaram o tema, quais docentes são especialistas na área e caracterizar a dinâmica de participação em bancas; apontar quais palavras-chave são utilizadas com mais frequência; bem como conhecer os avanços sobre o tema e classificar os trabalhos obtidos, a partir de revisão sistemática da literatura.

A revisão de literatura trata de um olhar crítico sobre as pesquisas existentes que são significantes para o desenvolvimento de um trabalho. A fundamentação teórica permite averiguar trabalhos e pessoas que estudaram sobre seu tema de pesquisa, que possibilita identificações de informações úteis para o desenvolvimento do trabalho, como palavras-chave e assuntos-chave (TURRIONI \& MELLO, 2012).

Em linhas gerais, existem três tipos de revisão de literatura: revisão narrativa, revisão sistemática e revisão integrativa, que podem apresentar ramificações (GRANT \& BOOTH, 2009). A revisão narrativa não utiliza critérios explícitos e sistemáticos para a busca e análise da literatura, nem aplica estratégias sofisticadas de busca. À medida que a revisão integrativa busca examinar rigorosamente e combinar estudos com diferentes princípios metodológicos, tem a capacidade de elaborar estudos de revisão em diversas áreas do conhecimento, mantendo a robustez metodológica das revisões sistemáticas (MATTOS, 2015).

No que se refere à revisão sistemática, esta começou a ser aplicada

\section{MATERIAL E MÉTODOS}

O mapeamento sistemático da literatura promove uma visão geral da área de pesquisa, permitindo identificar quantidades, tipos de pesquisas e seus resultados, e adicionalmente busca-se por profissionais da área da saúde, principalmente da medicina, a partir da década de 1980, e consiste em meio de identificar, avaliar e interpretar todas as pesquisas disponíveis importantes para a questão de pesquisa específica, área temática ou fenômeno de interesse. Esse tipo de revisão orienta o processo de investigação, evitando duplicidades de esforços e minimizando os erros, sendo cada vez mais importante diante do panorama atual de crescimento e disponibilidade de publicações científicas (KITCHENHAM, 2004; BIOLCHINI et al., 2005; FELIZARDO \& MARTINS, 2009).

Segundo Grant e Booth (2009) e Sousa et al. (2018), as revisões de literatura possuem várias ramificações considerando seu tipo: Revisão crítica; Revisão integrativa; Revisão de literatura; Revisão de mapeamento ou mapa sistemático; Meta análise; revisão de estudos mistos; Visão geral; Revisão sistemática qualitativa ou síntese de evidências qualitativas; Revisão rápida; Revisão scoping; Revisão do estado da arte; Revisão sistemática e pesquisa; Revisão sistematizada; e Revisão guardachuva ou de cobertura.

Diante do exposto e dos objetivos a serem alcançados, este trabalho possui natureza exploratória-descritiva. É exploratória, pois tem caráter preliminar e busca levantar mais informações sobre o tema da pesquisa - filtração lenta; e descritiva porque permite identificar características importante desta área de conhecimento, envolve o uso de técnicas padronizadas de coleta de dados, como mapeamento sistemático da literatura (PRODANOV \& FREITAS, 2013).

conhecer a frequência de publicações ao longo do tempo, a fim de se identificar tendências (PETERSEN et al., 2008). O mapeamento sistemático da literatura (MSL) baseia-se em um protocolo de 
pesquisa que aborda itens como: objetivo de busca, palavras-chave, mecanismo de buscas, filtros e critérios de seleção e extração de dados (KITCHENHAM, 2004). A Figura 1 apresenta as ações metodológicas realizadas para a obtenção do MSL para o tema de pesquisa em questão: filtração lenta, adotando como trabalhos de interesse teses e dissertações apresentadas em programas de pósgraduação brasileiros.

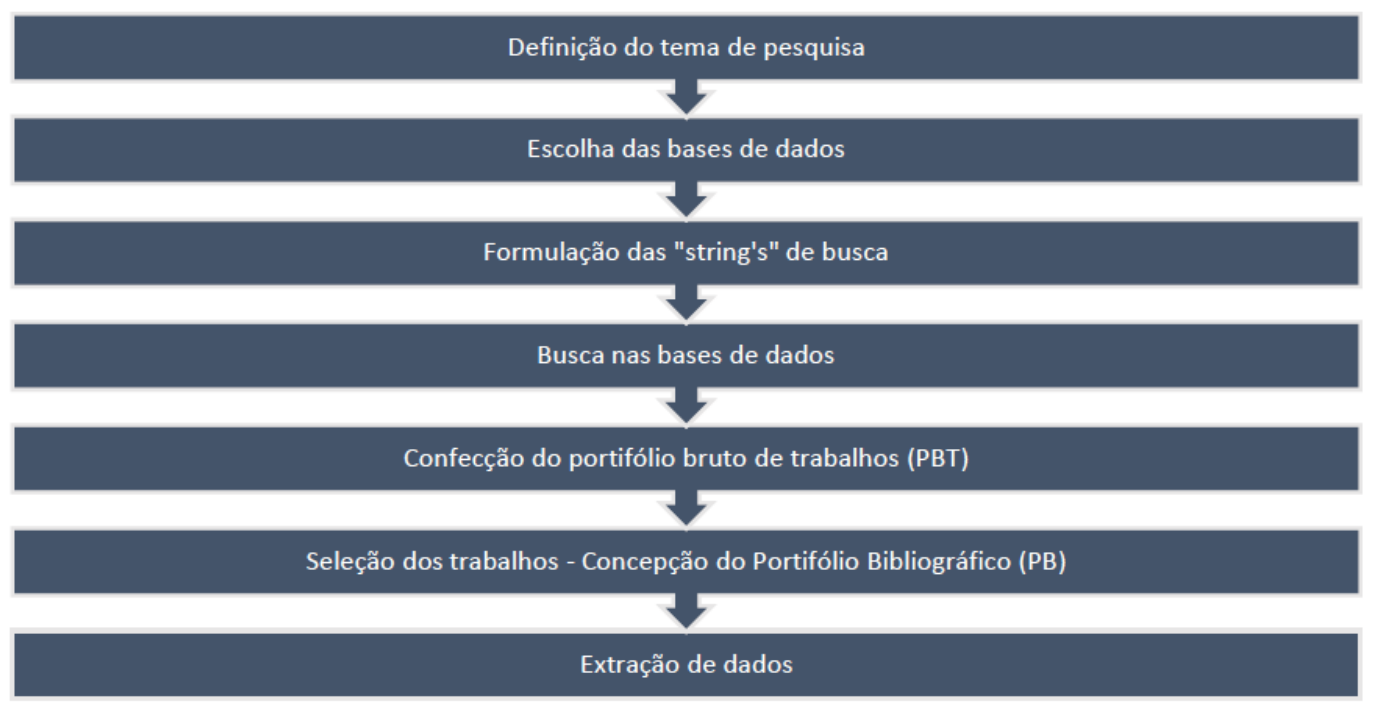

Figura 1: Passos para o MSL.

\subsection{Buscas no Catálogo da CAPES}

O mapeamento sistemático da literatura exige, de início, a definição dos termos de pesquisa com base no tema a ser estudado. Neste trabalho, foram utilizados os termos: (1) filtração lenta, (2) filtros lentos e (3) tratamento de água. O termo (2) foi utilizado no plural para que, durante a pesquisa, obtivesse trabalhos com termos tanto no plural quanto no singular. A adoção do termo (3) possibilitou restringir os resultados para aplicações em tratamento de água, tendo em vista que o processo de filtração lenta é aplicado, mas com menos frequência, no tratamento de efluentes. Nesta etapa não houve restrição de datas, sendo possível obter trabalhos dos mais antigos aos mais recentes.

Como este trabalho consiste em mapear sistematicamente os trabalhos de pós-graduação (dissertações e teses) brasileiros, utilizou-se como base de dados o Catálogo de Teses e Dissertações da Coordenação de Aperfeiçoamento de Pessoal de Nível Superior - CAPES. Este catálogo consiste em um sistema de busca bibliográfica, que reúne registros desde
1987, de teses e dissertações fornecidos à CAPES pelos programas de pósgraduação, que se responsabilizam pela veracidade das informações, onde é possível realizar a busca por autor, título e/ou palavra-chave (BRASIL, 2010).

Considerando as configurações e limitações da base de dados, formularamse duas strings de pesquisa:

- "filtração lenta" AND "tratamento de água";

- "filtros lentos" AND "tratamento de água".

As strings de busca são expressões formadas pelos termos de pesquisa e operadores (responsáveis pelo refino primário dos resultados de busca) suportados pela base de dados. Neste caso, os termos de pesquisa encontram-se entre aspas para que os registros obtidos na busca resultem em trabalhos que contenham, nos campos avaliados pela ferramenta de busca (autor, título e palavras-chave), exatamente os termos pesquisados. $\mathrm{O}$ operador AND restringe os registros encontrados a trabalhos que apresentem simultaneamente, nos campos avaliados, os dois termos de pesquisa. 
Além disso, limitou-se a exibição de resultados a trabalhos pertencentes à área de avaliação "Engenharias I", por meio da ferramenta "Refinar meus resultados".

Foi realizado o upload dos trabalhos obtidos a partir dos sites dos depositantes, normalmente repositórios institucionais, e suas informações foram catalogadas em planilha eletrônica para que fosse possível análises posteriores. Tendo observado a predominância de trabalhos com datas anteriores aos últimos 5 anos, optou-se por realizar buscas individualizadas nos repositórios de Instituições de Ensino Superior - IES, conforme metodologia descrita no próximo item, para avaliar o quão a base de dados utilizada estava atualizada em termos de trabalhos recentes.

\subsection{Buscas nos repositórios institucionais}

Diferentemente do item 2.1, nesta etapa foram realizadas buscas em repositórios de instituições de ensino superior, disponíveis digitalmente de forma individualizada, cujas bases de dados possam abrigar trabalhos relativos à filtração lenta, limitando os resultados por ano de publicação, seguindo os seguintes passos: identificação dos repositórios de pesquisa; busca na base de dados; e seleção dos trabalhos.

Para determinar quais IES teriam o tema de pesquisa buscado em seus respectivos repositórios eletrônicos, realizou-se o levantamento dos cursos de pós-graduação pertencentes à Área de Avaliação Engenharias I, mestrados ou doutorados acadêmicos, na Plataforma Sucupira, que é gerida pela CAPES. Uma vez identificadas as possíveis instituições

\section{RESULTADOS E DISCUSSÃO}

\subsection{Análise da busca individualizada nos repositórios}

A busca na Plataforma Sucupira resultou em 110 programas de pósgraduação pertencentes à área Engenharias que possuem trabalhos acerca do tema, buscou-se na internet os sites dos seus respectivos Repositórios Institucionais RI, catalogando-os para facilitar acessos futuros.

As buscas realizadas nos RI's tiveram como argumento de pesquisa a expressão "filtração lenta". Consideraramse trabalhos publicados no período de 2013 a 2018 (sendo realizadas as buscas em novembro de 2018), ou seja, os últimos 5 anos mais o ano corrente, e, sempre que possível, utilizaram-se os filtros correspondentes a "Assunto", disponíveis nos repositórios, para fazer convergir os resultados para trabalhos pertencentes a áreas relativas a Engenharias I. Os trabalhos encontrados tinham seus títulos lidos instrumentalmente e caso não houvesse aderência do título com o tema da pesquisa eram descartados.

No entanto, quando a pesquisa resultava em número elevado de trabalhos $\mathrm{e}$, adicionalmente, filtros de assuntos muitos pulverizados, dada às limitações do algoritmo de pesquisa dos respectivos RI's, de modo a tornar impraticável a leitura de todos os títulos, optou-se por ler os títulos de trabalhos que apresentavam palavras como: filtração, água e tratamento. Para tanto, utilizou a ferramenta do navegador "Localizar nesta página".

Os trabalhos obtidos nos itens 2.1 (busca no catálogo da CAPES sem limite de tempo) e 2.2 (busca individualizada nos RI com limite de tempo, para obter trabalhos atuais) formaram o Portifólio Bruto de Trabalho (PBT), que fora utilizado nas demais etapas do mapeamento sistemático apresentadas na Figura 1.

I, destes, 99 estão em funcionamento, $4 \mathrm{em}$ processo de desativação, 4 desativados e dois em projeto. Para fins de levantamento dos repositórios, foram consideradas somente as instituições que possuíam programas ativos. A maioria dos 
programas encontrados, tanto de mestrado quanto de doutorado, são de Engenharia Civil, seguidos de Engenharia Sanitária e Engenharia de Transportes. As Figuras $2 \mathrm{e}$ 3 ilustram aspectos quantitativos dos programas de pós-graduação incluídos na área de avaliação "Engenharias I", em termos de áreas básicas, IES, e Unidades da Federação - UF, para mestrado e doutorado, respectivamente.

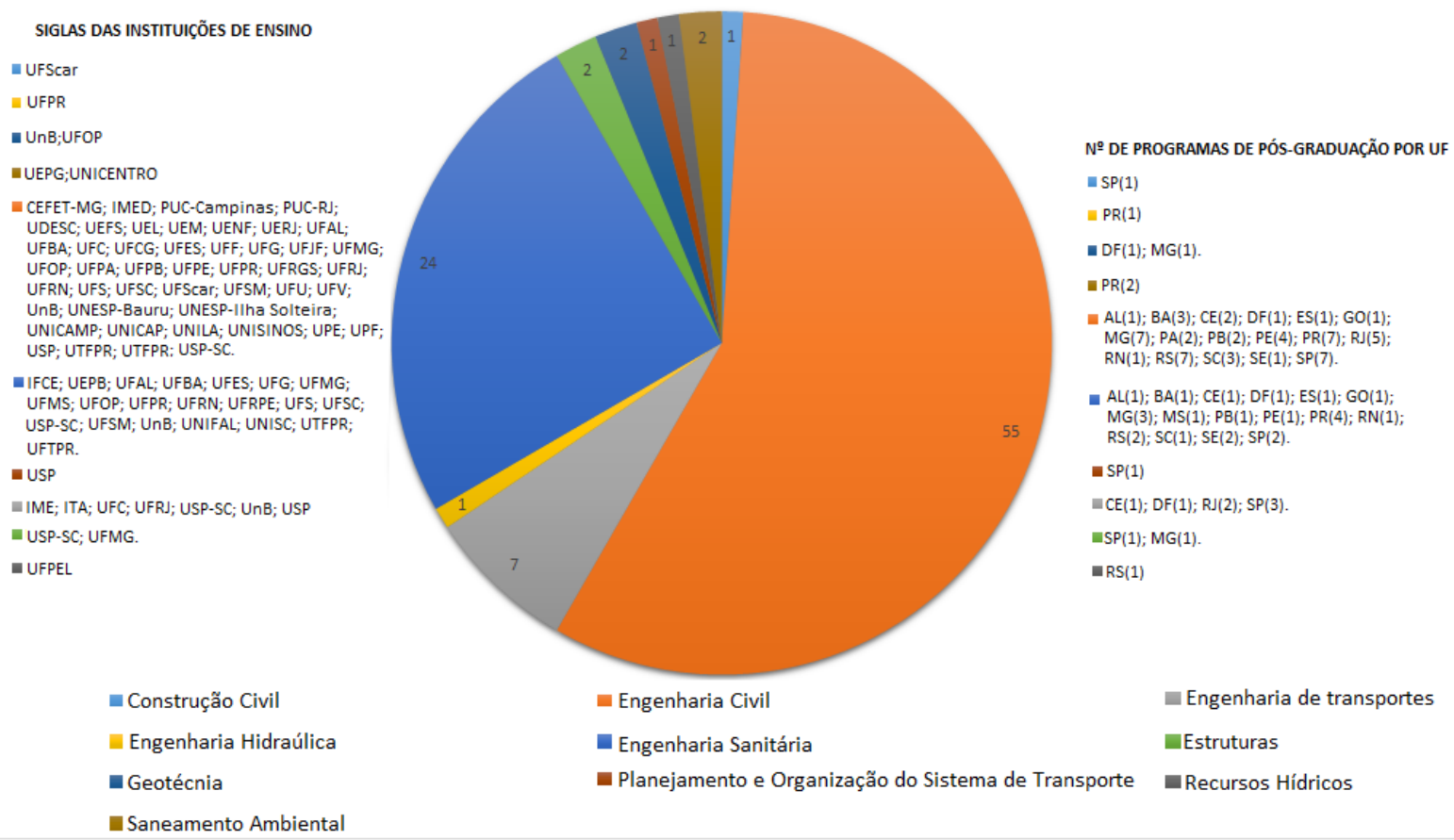

Figura 2: Cursos de mestrado por área básica.

SIGLAS DAS INSTITUIÇÕES DE ENSINO

=UFScar

- CEFET-MG; PUC-RJ; UEL; UENF; UERJ; UFAL; UFBA; UFC; UFCG; UFF; UFG; UFOP; UFPA; UFPB; UFPE; UFPR; UFRGS. UNESP UPF; USP; UTFPR; USP-SC.

=ITA; UFC; UFRJ; USP-SC; UnB; USP

$=U F P R$

-UEPB; UFES; UFMG; UFMS; UFOP; UFPR.

UFSC; USP-SC; UFSM; UnB; UNISC.

IUSP-SC; UFMG.

-UnB; UFOP.

- UFPEL

- UERJ

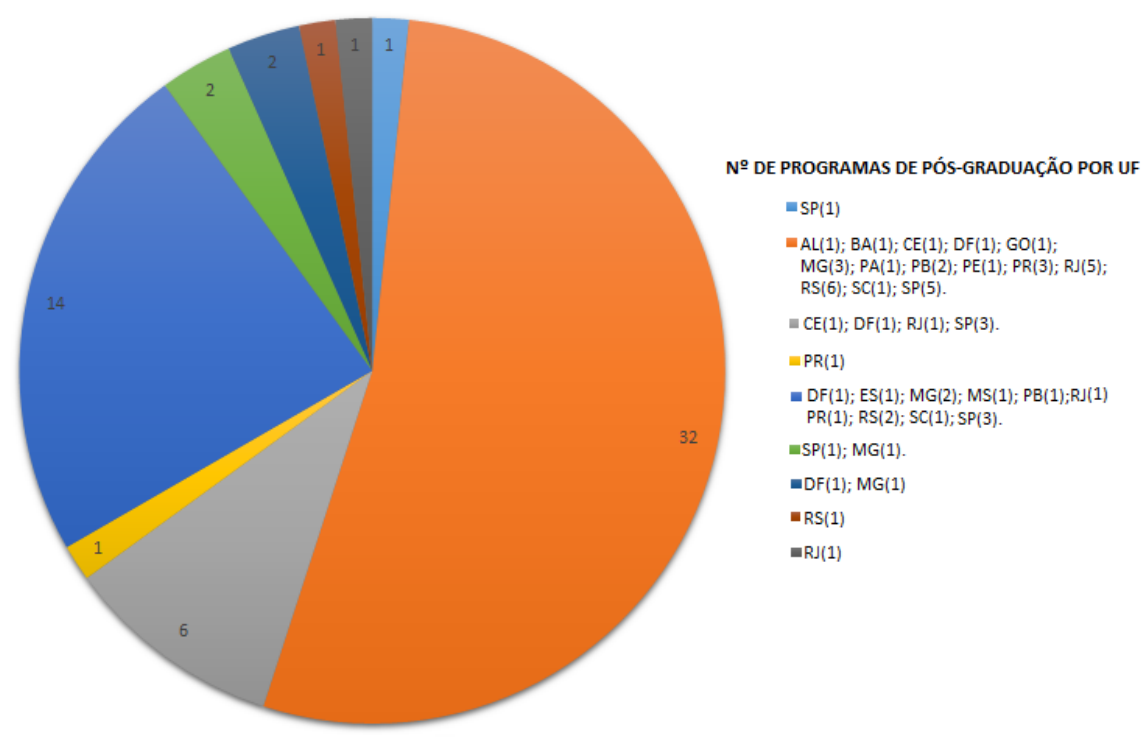

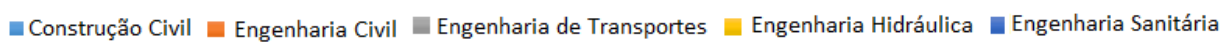

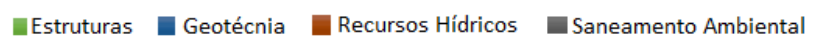

Figura 3: Cursos de doutorado por área básica. 
Das 55 IES apresentadas nas Figuras 1 e 2, 4 não tiveram seus repositórios encontrados eletronicamente. Dos 51 repositórios restantes apenas 40 apresentaram retornos para os argumentos de pesquisa, resultando no total de 10.928 trabalhos dos quais foram selecionados 11 , distribuídos entre duas instituições das 55 avaliadas, e incorporados aos trabalhos obtidos no Catálogo de Teses e Dissertações da CAPES, pois, a priori, apresentavam títulos de interessa para pesquisa.

\subsection{Portifólio bibliográfico e análise bibliométrica}

O portifólio bruto de trabalhos (PBT) consistia de 108 trabalhos, dentre teses e dissertações, resultado da soma dos 97 trabalhos obtidos no item 2.1 e 11 trabalhos selecionados no item 2.2. A fim de se obter trabalhos alinhados com o tema da pesquisa, submeteu-se o PBT a diversos filtros e critérios, para se obter um portifólio bibliográfico $(\mathrm{PB})$ enxuto e consistente. $\mathrm{Na}$ Figura 4, podem-se observar esses critérios e filtros, bem como o número de trabalhos obtidos após cada um.

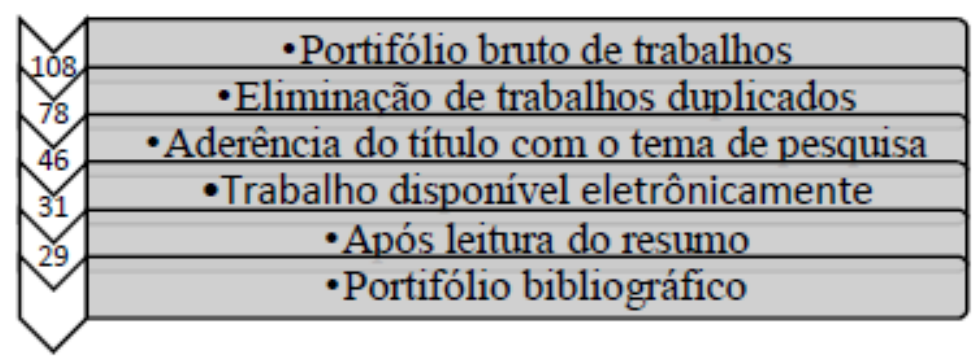

Figura 4: Metodologia para concepção do Portifólio Bibliográfico.

Ao fim do processo de busca, foram selecionados 29 trabalhos para compor o PB, sendo 20 dissertações de mestrado e 9 teses de doutorado. O Quadro 1 apresenta estes trabalhos classificados por autor, ano de publicação em ordem cronológica, tipo de trabalho (mestrado ou doutorado) e instituição de origem.

Quadro 1: Trabalho selecionados para compor o Portifólio Bibliográfico.

\begin{tabular}{|c|c|c|c|c|c|}
\hline & Título & Tipo* & Autor & Ano & Instituição \\
\hline 1 & $\begin{array}{l}\text { Avaliação do desempenho da filtração da } \\
\text { filtração em múltiplas etapas no tratamento } \\
\text { de águas com elevadas concentrações de } \\
\text { algas. }\end{array}$ & $\mathrm{D}$ & Mello, O. M. T. & 1998 & UnB \\
\hline 2 & $\begin{array}{l}\text { Influência da redução da espessura da } \\
\text { camada suporte na eficiência da filtração } \\
\text { lenta com uso de mantas sintéticas não } \\
\text { tecidas para águas de abastecimento. }\end{array}$ & $\mathrm{D}$ & Ferraz, C. F. & 2001 & UNICAMP \\
\hline 3 & $\begin{array}{l}\text { Influência da pré-oxidação com ozônio e } \\
\text { peróxido de hidrogênio no desempenho da } \\
\text { filtração lenta em areia e carvão ativado } \\
\text { granular. }\end{array}$ & $\mathrm{D}$ & $\begin{array}{l}\text { Aguila, N. K. S. } \\
\text { D. }\end{array}$ & 2002 & USP \\
\hline 4 & $\begin{array}{c}\text { Influência da pré-oxidação com ozônio e } \\
\text { peróxido de hidrogênio na remoção de } \\
\text { atrazina em filtros lentos de areia e carvão } \\
\text { ativado granular. }\end{array}$ & $\mathrm{T}$ & Coelho, E. R. C. & 2002 & USP \\
\hline 5 & $\begin{array}{l}\text { Remoção de Microcystis aeruginosa e } \\
\text { microcistina pelo processo de filtração lenta. }\end{array}$ & $\mathrm{D}$ & Sá, J. C. & 2002 & UnB \\
\hline 6 & $\begin{array}{l}\text { Remoção de substâncias húmicas por meio } \\
\text { da oxidação com ozônio e peróxido de } \\
\text { hidrogênio e filtração lenta. }\end{array}$ & $\mathrm{T}$ & Tangerino, E. P. & 2003 & USP \\
\hline
\end{tabular}




\begin{tabular}{|c|c|c|c|c|c|}
\hline 7 & $\begin{array}{l}\text { Remoção do indicador Clostridium } \\
\text { perfringens e de oocistos de } \\
\text { Cryptosporidium parvum por meio da } \\
\text { filtração lenta - Avaliação em escala piloto. }\end{array}$ & $\mathrm{D}$ & Peralta, C. C. & 2005 & UnB \\
\hline 8 & $\begin{array}{l}\text { Avaliação da filtração lenta na Remoção de } \\
\text { células de Cylindrospermopsis raciborskii e } \\
\text { saxitoxinas. }\end{array}$ & $\mathrm{D}$ & Melo, A. E. S. & 2006 & UnB \\
\hline 9 & $\begin{array}{l}\text { Influência das características da camada } \\
\text { filtrante e da taxa de filtração na eficiência } \\
\text { de remoção de Microcystis aeruginosa e } \\
\text { microcistina na filtração lenta em areia. }\end{array}$ & $\mathrm{T}$ & Sá, J. C. & 2006 & UnB \\
\hline 10 & $\begin{array}{l}\text { Tratamento de água de chuva através de } \\
\text { filtração lenta e desinfecção UV. }\end{array}$ & $\mathrm{D}$ & Bastos, F. P. & 2007 & UFES \\
\hline 11 & $\begin{array}{l}\text { Remoção de oocistos de Cryptosporidium } \\
\text { na filtração lenta precedida ou não de } \\
\text { filtração ascendente em pedregulho. }\end{array}$ & $\mathrm{D}$ & Taira, R. & 2008 & UnB \\
\hline 12 & $\begin{array}{l}\text { Utilização de colunas verticais de filtração } \\
\text { em manta e areia como pré-tratamento de } \\
\text { filtro lento. }\end{array}$ & $\mathrm{D}$ & Tavares, M. B. & 2008 & UNESP \\
\hline 13 & $\begin{array}{l}\text { Desempenho da Filtração em Múltiplas } \\
\text { Etapas (FiME) no tratamento de águas de } \\
\text { abastecimento em escala piloto. }\end{array}$ & $\mathrm{D}$ & Camplesi, D. C. F. & 2009 & UFG \\
\hline 14 & $\begin{array}{l}\text { Avaliação do desempenho da filtração em } \\
\text { múltiplas etapas (FiME) no tratamento da } \\
\text { água do Ribeirão Sozinha em Goianápolis - } \\
\text { Estado de Goiás. }\end{array}$ & $\mathrm{D}$ & Perez, W. E. & 2009 & UFG \\
\hline 15 & $\begin{array}{l}\text { Filtração em margem de rio precedendo a } \\
\text { filtração lenta para remoção de carbofurano, } \\
\text { em tratamento de água para consumo } \\
\text { humano. }\end{array}$ & $\mathrm{T}$ & $\begin{array}{l}\text { Michelan, D. C. } \\
\text { G. S. }\end{array}$ & 2010 & UFSC \\
\hline 16 & $\begin{array}{c}\text { Estudos de limpeza de filtro lento por } \\
\text { raspagem e retrolavagem. }\end{array}$ & $\mathrm{D}$ & Pizzolatti, B. S. & 2010 & UFSC \\
\hline 17 & $\begin{array}{l}\text { Remoção de micropoluentes na filtração } \\
\text { lenta com pré-oxidação com radiação solar. }\end{array}$ & $\mathrm{D}$ & Rossi, C. H. & 2010 & UNESP \\
\hline 18 & $\begin{array}{c}\text { Desempenho da filtração lenta com } \\
\text { diferentes períodos de amadurecimento no } \\
\text { tratamento de águas contendo elevadas } \\
\text { densidades de células tóxicas Microcystis } \\
\text { aeruginosa. }\end{array}$ & $\mathrm{D}$ & Salati, D. S. & 2010 & UnB \\
\hline 19 & $\begin{array}{l}\text { Estudo da remoção do herbicida atrazina por } \\
\text { biofiltração em filtros lentos de areia e } \\
\text { carvão ativado associada à ação microbiana. }\end{array}$ & $\mathrm{D}$ & Zanini, J. & 2010 & UNESP \\
\hline 20 & $\begin{array}{l}\text { Desempenho de filtros lentos, com } \\
\text { diferentes períodos de amadurecimento, } \\
\text { precedidos de pré-filtração em pedregulho } \\
\text { no tratamento de águas contendo células } \\
\text { tóxicas de Microcystis aeruginosa. }\end{array}$ & $\mathrm{D}$ & Farias, N. J. V. & 2011 & UnB \\
\hline 21 & $\begin{array}{c}\text { Avaliação da qualidade da água de chuva } \\
\text { em Maceió/AL coletada em telhados: } \\
\text { tratamento através de filtração lenta e } \\
\text { possíveis utilizações. }\end{array}$ & $\mathrm{D}$ & Souza, V. C. & 2011 & UFAL \\
\hline 22 & $\begin{array}{l}\text { Influência da técnica de limpeza de filtros } \\
\text { lentos e diâmetro dos grãos sobre a } \\
\text { qualidade da água produzida, com destaque } \\
\text { para remoção de carbamatos e oocistos de }\end{array}$ & $\mathrm{T}$ & Pizzolatti, B. S. & 2014 & UFSC \\
\hline
\end{tabular}




\section{Cryptosporidium (simulados por microesferas).}

Redução da concentração de herbicidas em águas por filtração em múltiplas etapas

23 seguida de coluna de carvão ativado de babaçu e obtenção de adsorventes a partir de bagaço de cana e casca de Moringa oleifera. Remoção de metais pesados da água por

24 filtração lenta em não tecidos com auxílio de coagulante extraído de sementes de 'Moringa oleifera'.

Comparação entre os meios filtrantes areia e

25 não tecido sintético na eficiência da filtração lenta para tratamento de água.

Tratamento de água para Abastecimento por meio de Filtros Lentos de Fluxo Ascendente com Limpeza por Retrolavagem e Descarga de Fundo.

Influência de Moringa oleifera L. na

27 remoção de cianobactérias na filtração lenta com manta não tecida.

Filtros lentos em escala domiciliar como

alternativa de tratamento de águas com alto risco microbiológico em comunidades isoladas.

Desempenho de coagulantes naturais adsorvente alternativo e filtros lentos 29 domiciliares no tratamento de águas com microcystis aeruginosa e microcistina: alternativas tecnológicas para comunidades isoladas.

\begin{tabular}{|c|c|c|c|}
\hline T & Zoratto, A. C. & 2014 & UNICAMP \\
\hline T & Franco, M. & 2015 & UNICAMP \\
\hline T & Santos, F. F. S. & 2015 & UNICAMP \\
\hline D & Souza, F. H. & 2015 & UFSC \\
\hline D & Barros, S. G. B. & 2017 & UNICAMP \\
\hline & Freitas, B. L. S. & 2017 & USP \\
\hline
\end{tabular}

Como base nos trabalhos selecionados, fez-se um estudo das palavras-chave mais utilizadas para verificar a aderência das palavras usadas nas strings de busca com as comumente

utilizadas em trabalhos a respeito do tema de pesquisa. A Figura 5 exibe as palavraschave utilizadas nos trabalhos do PB. Quanto maior é o tamanho da fonte, maior é a frequência com que fora utilizada.

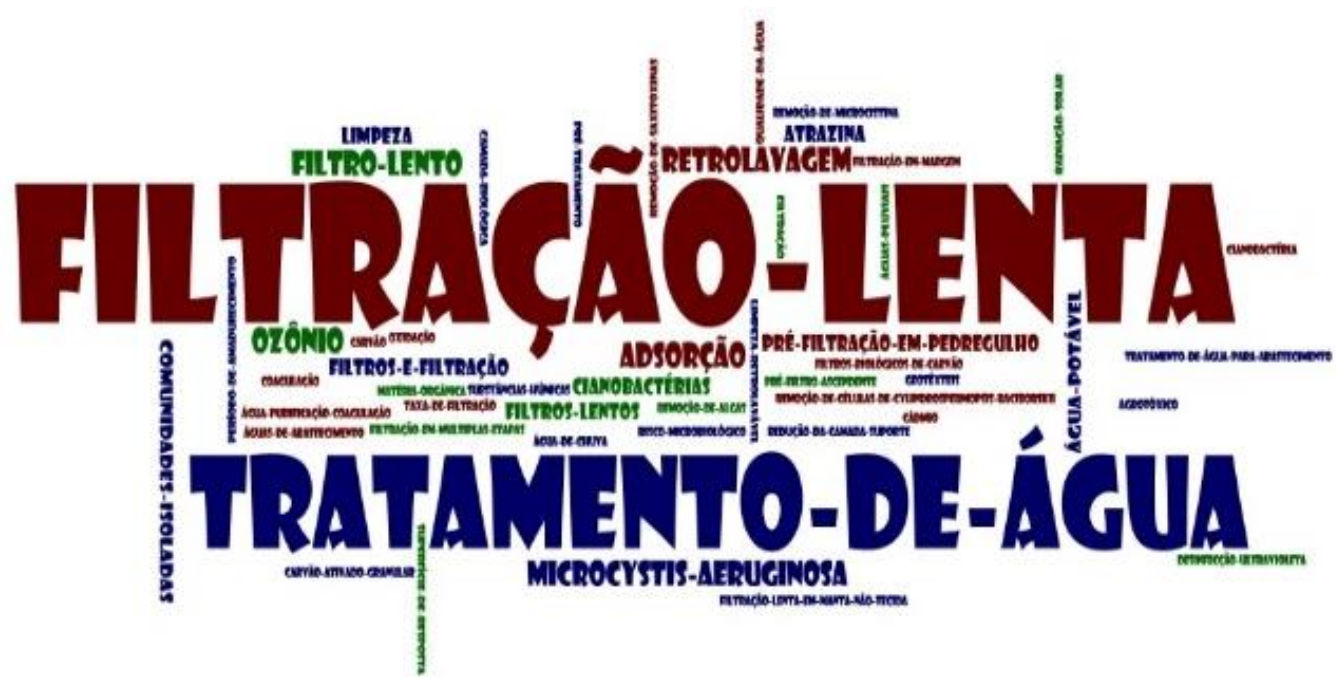

Figura 5: Principais palavras-chave utilizadas. 
Dos três termos utilizados na pesquisa, dois são os mais utilizados como palavras-chave. Logo, os termos "filtração lenta" e "tratamento de água" são indicados para buscas sobre o tema da pesquisa. As demais palavras utilizadas são termos inerentes a algum aspecto da filtração lenta: tipo de pré ou póstratamento, parâmetros de controle da qualidade da água, operação etc.
A maior parte das dissertações de mestrado é oriunda da Universidade de Brasília (UnB) - 7 trabalhos, enquanto que, em relação às teses de doutorado, a Universidade Estadual de Campinas (UNICAMP) se destaca com 4 trabalhos. $\mathrm{Na}$ Figura 6, é exibido o panorama de publicações nos últimos 20 anos, com destaque para 2010, com o maior número de publicação sobre o tema.

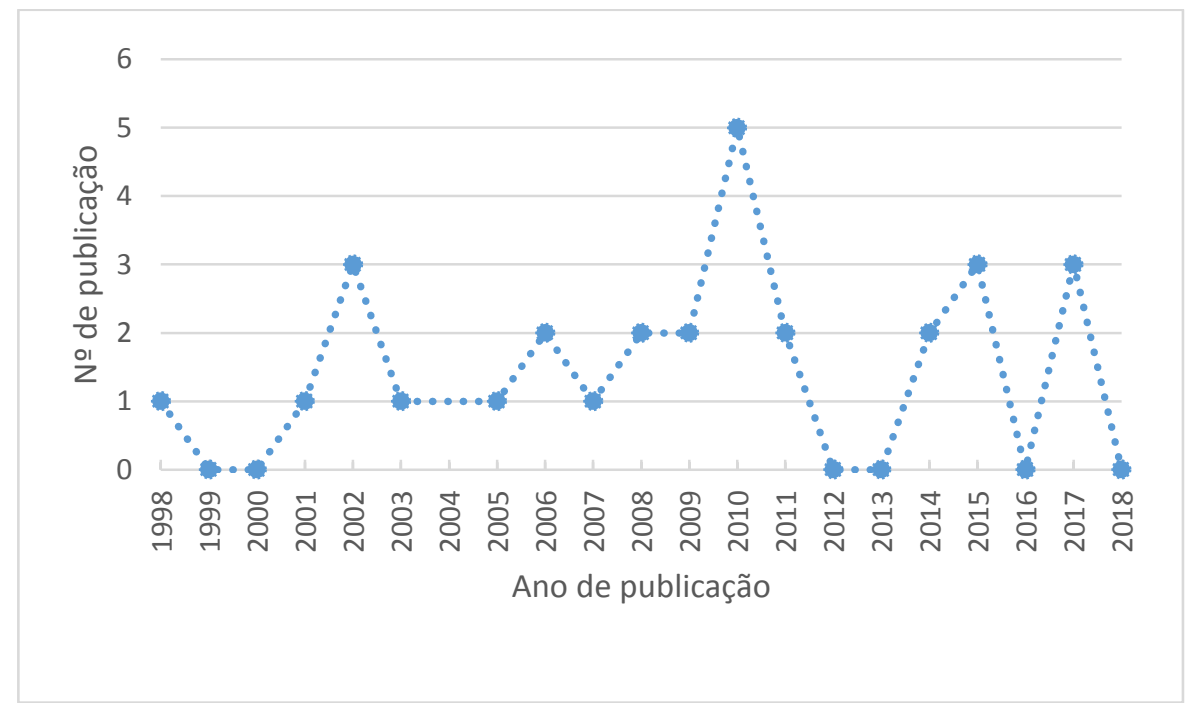

Figura 6: Número de publicação por período.

Nos últimos 20 anos, houve ausência de trabalhos a respeito da filtração lenta somente nos anos de 1999, 2000, 2012, 2013 e 2016. Apesar do ano de 2018 não apresentar nenhum trabalho publicado não pode ser considerado ausente de trabalhos, tendo em vista que é o ano corrente na data de desenvolvimento deste trabalho e que pode vir a ter alguma publicação até seu término.

A fim de se verificar quais IES apresentam maior volume de trabalhos publicados acerca do tema de pesquisa, foi elaborado um gráfico de barras contendo o número de trabalhos desenvolvidos em cada instituição (Figura 7).

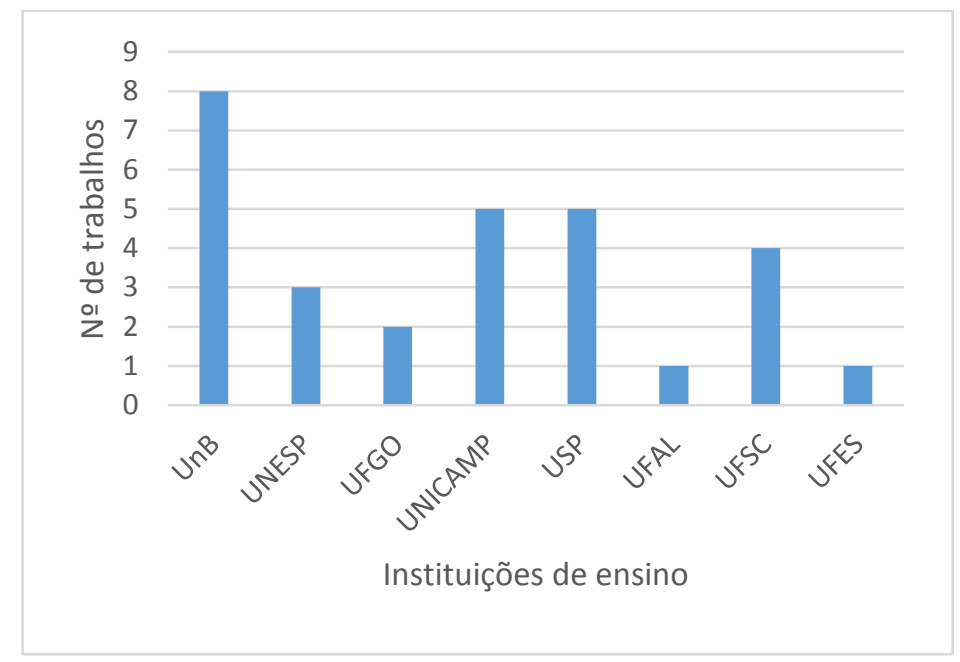

Figura 7: Publicações por instituição de ensino. 
Como pode ser observado, a UnB é responsável pelo maior número de trabalhos publicados no período em estudo, seguida da UNICAMP e Universidade de São Paulo (USP). É importante destacar a hegemonia das regiões centro-oeste, sudeste e sul na produção de trabalhos sobre filtração lenta. Com exceção do trabalho publicado na Universidade Federal de Alagoas (UFAL), todos pertencem a área formada por essas três regiões, sendo a UnB, UNICAMP e USP responsáveis por mais de $60 \%$ das publicações. O que está em consonância com o fato de que a maioria dos livros brasileiros sobre tratamento de água e que abordam filtração lenta têm seus autores vinculados a alguma instituição dessas regiões.

Em se tratando de teses e dissertação, faz-se necessário associar os trabalhos publicados não tão somente aos autores como também aos respectivos orientadores, pois suas contribuições são fundamentais para o desenvolvimento da pesquisa, principalmente quando estes são referências na área em estudo. Para avaliar quem são os docentes que mais orientam trabalhos que abordam a temática desta pesquisa elaborou-se o gráfico da Figura 8 .

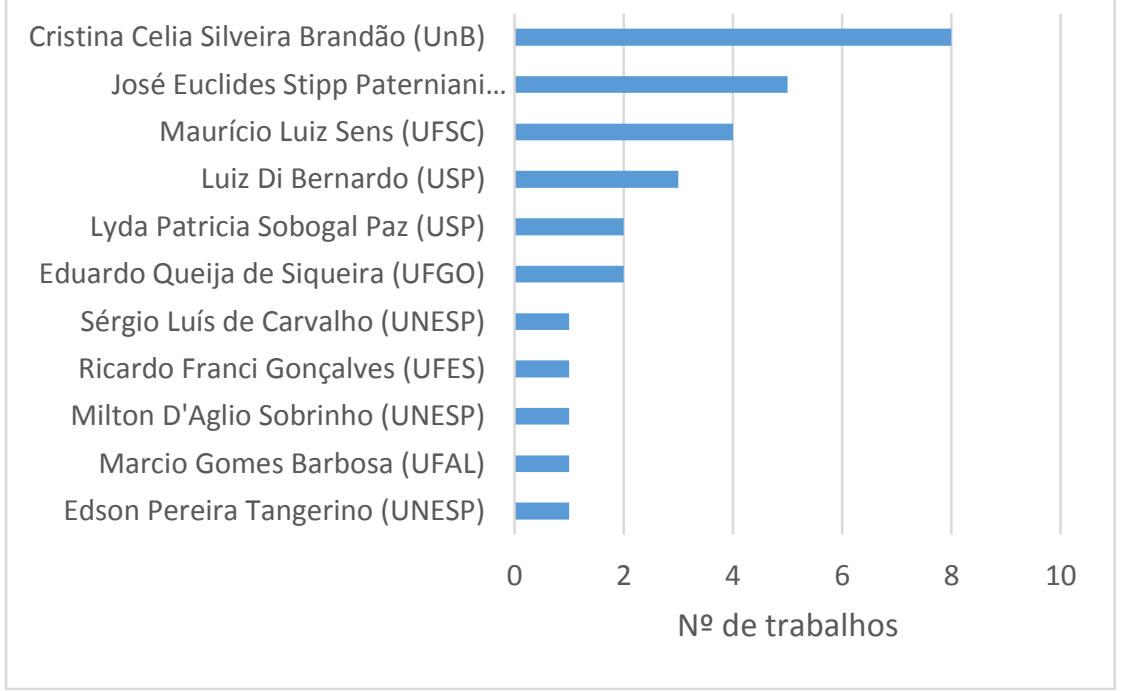

Figura 8: Número de orientações.

No âmbito das orientações de trabalhos sobre filtração lenta, destaca-se a Prof $^{a}$ Cristina Célia Silveira Brandão, da UnB, com a orientação em 8 trabalhos, que representa a totalidade de trabalhos oriundos da UnB. Na sequência, têm-se o Prof. José Euclides Stipp Paterniani da UNICAMP, Prof. Maurício Luiz Sens da Universidade Federal de Santa Catarina (UFSC) e Luiz Di Bernardo da USP, com 5,4 e 3 orientações, respectivamente. A Prof. ${ }^{a}$ Cristina Célia Silveira Brandão foi quem mais orientou dissertações de mestrado (7 trabalhos), enquanto o Prof. José Euclides Stipp Paterniani foi quem mais orientou teses de doutorados (4 trabalhos).

Este trabalho também avaliou a dinâmica de participação em bancas, a fim de visualizar, principalmente, o grau de comunicação entre professoresorientadores. A Figura 9 apresenta esse grau de intercomunicação dos envolvidos. Em negrito estão os nomes dos orientadores, enquanto que o sentido da seta indica o convite para participação da banca. Os números contidos nas setas e as cores das linhas (linha preta: 1 convite, linha vermelha: 2 convites, linha azul: 3 convites e linha verde: 4 convites) indicam a quantidade de convites.

$\mathrm{Na}$ Figura 10, é possível observar que, com exceção de três professores, Lyda Patrícia Sabogal Paz, Ricardo Franci Gonçalves e Márcio Gomes Barboza, todos orientadores apresentam algum tipo de relação entre si, seja como anfitrião ou convidado. Nesta dinâmica, destaca-se o 
Prof. Luiz Di Bernardo como quem mais convidou professores-orientadores para banca de seus alunos, de maneira que o Prof. Edson Pereira Tangerino foi $o$ professor-orientador que mais participou de banca como convidado. Outros professores também receberam quantidade significativa de convites, mas a maioria é da mesma instituição dos professoresorientadores, o que justifica tal comportamento.

Por fim, classificou-se os trabalhos acerca da filtração lenta quanto ao seu enfoque, sentido do fluxo, taxa de filtração, tipos de pré e pós-tratamentos e leito filtrante. A Figura 9 demonstra esse agrupamento, em que os números entre parênteses se referem à relação de trabalhos do Quadro 1.

A partir da Figura 9, pode-se constatar que a maior parte dos trabalhos possui uma perspectiva a respeito da eficiência do tratamento. Em geral, os trabalhos que adotaram taxa de filtração de $3 \mathrm{~m}^{3} / \mathrm{m}^{2}$.dia, tendiam a não utilizar prétratamento e/ou pós-tratamento, apresentando, corriqueiramente, em seus aspectos metodológicos, filtros lentos de areia convencionais, seja como unidade principal de estudo ou como unidade de controle.

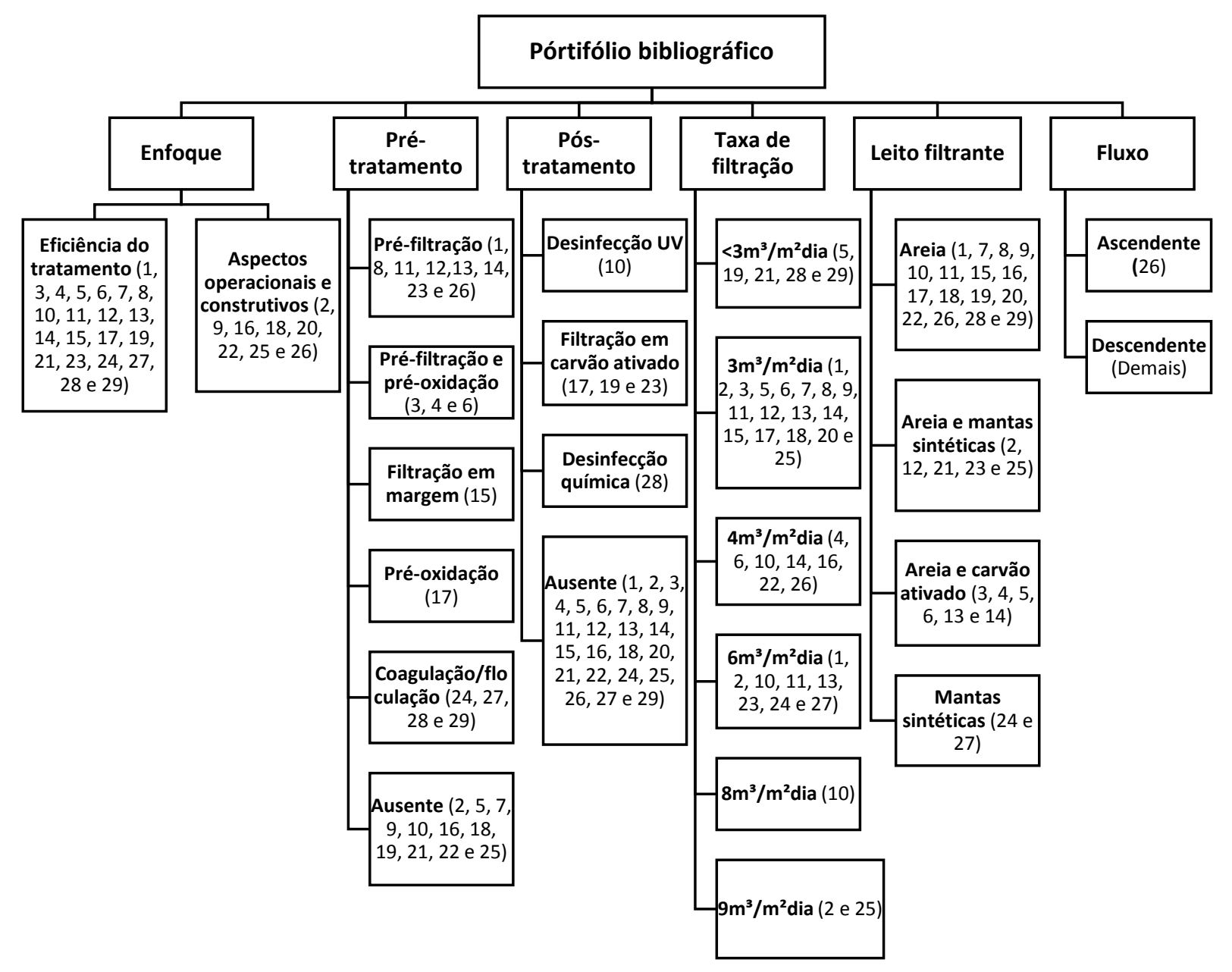

Figura 9: Classificação dos trabalhos do PB. 


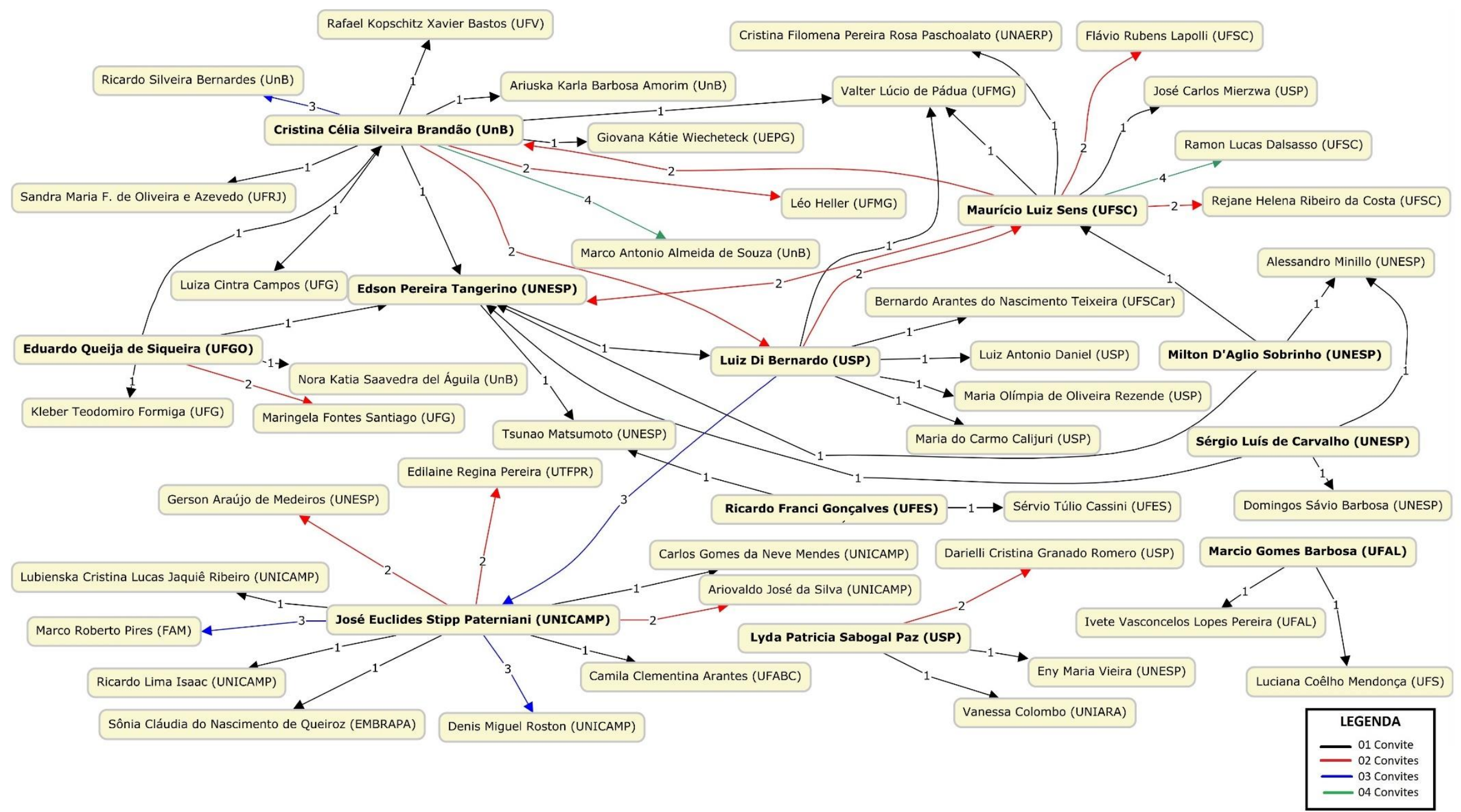

Figura 10: Panorama de participação em bancas. 


\section{CONCLUSÕES}

Com este trabalho, observou-se que a maioria de trabalhos sobre filtração lenta foi desenvolvida na UnB. Esta instituição também se destaca quanto à produção de dissertações de mestrado, enquanto a UNICAMP é responsável pela maior quantidade de produção de teses de doutorado. Constatou-se uma concentração regional das pesquisas sobre o tema formada pelas regiões sudeste, centro-oeste e sul, que detêm quase a totalidade das pesquisas realizadas. Além disso, há boa relação entre a maioria dos pesquisadores lotados nas instituições de ensino nessas regiões, notabilizadas pelo número de convites e participação de bancas em trabalhos sobre o tema.

Contabilizaram-se 29 publicações acerca do tema nos últimos 20 anos, sendo a maior produção no ano de 2010. Juntas, a UnB, USP e UNICAMP englobam mais de

\section{AGRADECIMENTOS}

O presente trabalho foi desenvolvido com o apoio da Coordenação de Aperfeiçoamento de Pessoal de Nível

\section{REFERÊNCIAS BIBLIOGRÁFICAS}

BIOLCHINI, J. et al. Systematic Review in Software Engineering. Rio de Janeiro: COPPE/UFRJ, 2005.

BRASIL, M. da E. CAPES. Site Institucional. 2010. Disponível em: <http://www.capes.gov.br/acessoainformac ao/perguntas-frequentes/periodicos/3571como-funciona-o-banco-de-teses $>$. Acesso em: 20 nov. 2018.

BRITO, L. L. A. de et al. Amadurecimento de filtros lentos de areia e remoção de microrganismos indicadores de qualidade da água ao longo da profundidade do leito: uma avaliação em instalação piloto. Engenharia Sanitária e Ambiental, [s. 1.], v. 10, n. 4, p. 307-317, 2005.
$60 \%$ dos trabalhos, sendo esta última a instituição que mais publicou trabalhos de dissertação ou tese nos últimos 5 anos. Adicionalmente, este trabalho permitiu identificar e quantificar os programas de pós-graduação pertencente à área "Engenharias I", representando uma fonte de informação útil para os estudantes da área.

Por fim, este trabalho se faz útil para os pesquisadores da filtração lenta, principalmente para os estudantes de pósgraduação, pois apresentam um panorama dos principais trabalhos desenvolvidos pelos pesquisadores brasileiros, bem como demonstra a eficiência dos termos de pesquisa utilizados para busca em base de dados. Recomenda-se que, em casos de busca com aspectos mais específicos sobre o tema de pesquisa, sejam adicionados novos termos de pesquisa.

Superior - Brasil (CAPES) - Código de financiamento 001.

DI BERNARDO, L. (ED.). Tratamento de água para abastecimento por filtração direta. São Carlos: RiMa, 2003.

DI BERNARDO, L.; DANTAS, A. D. B. Métodos e técnicas de tratamento de água. $2^{\circ}$ ed. São Paulo: RiMa, 2005. v. 2.

DI BERNARDO, L.; SOBOGAL PAZ, L. $P$. Seleção de tecnologia de tratamento de água. São Carlos: LDIBE LTDA, 2008.

FELIZARDO, K. R.; MARTINS, R. M. Engenharia de Software Experimental: Revisão Sistemática, ICMC/USP, 2009.

GRANT, M. J.; BOOTH, A. A typology of reviews: an analysis of 14 review types and associated methodologies. Health Information and Libraries Journal, [s. 1.], v. 26, n. 2, p. 91-108, 2009. 
HELLER, L. et al. Oocistos de Cryptosporidium e cistos de Giardia: circulação no ambiente e riscos à saúde humana. Epidemiologia e Serviços de Saúde, [s. 1.], v. 13, n. 2, p. 79-92, 2004.

KITCHENHAM, B. Procedures for Performing Systematic Reviews. Keele: University Keele, 2004.

MATTOS, P. de C. Tipos de revisão da literatura, UNESP, 2015. Disponível em: <http://www.fca.unesp.br/Home/Biblioteca /tipos-de-evisao-de-literatura.pdf $>$. Acesso em: 28 nov. 2018.

PETERSEN, K. et al. Systematic Mapping Studies in Software Engineering. 12th International Conference on Evaluation and Assessment in Software Engineering, [s. 1.], 2008. Disponível em: <http://www.robertfeldt.net/publications/p etersen_ease 08_sysmap_studies_in_se.pdf >. Acesso em: 29 nov. 2018.

PRODANOV, C. C.; FREITAS, E. C. de. Metodologia do trabalho científico [recurso eletrônico]: métodos e técnicas da pesquisa e do trabalho acadêmico. $2^{\circ}$ ed. Novo Hamburgo: Feevale, 2013.
RICHTER, C. A. Água: métodos e tecnologia de tratamento. São Paulo: Blucher, 2009.

SOUSA, L. M. M. et al. Revisão da literatura científica: tipos, métodos e aplicações em enfermagem. RPER número O, [s. 1.], 2018.

SOUZA, F. H. de et al. Diagnóstico e discussão sobre uso da Filtração Lenta para abastecimento público em Santa Catarina, Brasil. Revista DAE, [s. 1.], v. 66, n. 209, p. 37-50, 2018.

TURRIONI, J. B.; MELlO, C. H. P. Metodologia de Pesquisa em Engenharia de Produção, Universidade Federal de Itajaí - UNIFEI, 2012.

VARGAS, L. C. Filtración lenta y dinámica como desinfectantes. In: Simposio regional sobre calidad del água: desinfección efectiva. Lima, 1999. Anais.

Disponível em: <https://www.itacanet.org/esp/agua/Se ccion $\% 206 \% 20$ Tratamiento\%20de $\% 20$ agu a/introduccionfiltracionarena.pdf $>$. Acesso em: 1 dez. 2018. 